\title{
Marginal Likelihood-based One-sided LR Test for Testing Higher Order Autocorrelation in Presence of Nuisance Parameters -A Distance Based Approach
}

\author{
Rumana Rois ${ }^{1}$, Tapati Basak $^{1}$ \& Ajit K. Majumder ${ }^{1}$ \\ ${ }^{1}$ Department of Statistics, Jahangirnagar University, Savar, Dhaka, Bangladesh \\ Correspondence: Rumana Rois, Department of Statistics, Jahangirnagar University, Savar, Dhaka-1342, Bangladesh. \\ E-mail: rumana248@yahoo.com
}

Received: June 15, 2012 Accepted: July 3, 2012 Online Published: July 30, 2012

doi:10.5539/ijsp.v1n2p69 URL: http://dx.doi.org/10.5539/ijsp.v1n2p69

\begin{abstract}
In most of the cases, only a subvector of the parameters is tested in a model. The remaining parameters arise in the tests as nuisance parameters. The presence of nuisance parameters causes biases in key estimates used in the tests. So inferences made on the presence of nuisance parameters may lead to less accurate conclusions. Even the presence of nuisance parameters can destroy the test. Thus in eliminating the influence of nuisance parameters from the test can improve the tests' performance. The effect of the nuisance parameters can be eliminated by the marginal likelihood, conditional likelihood, canonical likelihood, profile likelihood and Bayesian tests. This paper is concerned with marginal likelihood-based test for eliminating the influence of nuisance parameters. In general, existing one-sided and two-sided tests for autocorrelation are tested only autocorrelation coefficients but not the regression coefficients in the model. So we proposed a distance-based marginal likelihood one-sided Likelihood Ratio (DMLR) test in eliminating the influence of nuisance parameters for testing higher order autocorrelation with one-sided alternatives in linear regression model using marginal likelihood and distance-based approach. Monte Carlo simulations are conducted to compare power properties of the proposed DMLR test with their respective conventional counterparts. It is found that the DMLR test shows substantially improved power for most of cases considered.
\end{abstract}

Keywords: distance-based likelihood ratio test, Monte Carlo simulation, one-sided alternatives, power

JEL Classification Code: C12, C15, C22

\section{Introduction}

The inference in the presence of nuisance parameters (the parameters not under test) is a long-standing problem in econometrics as well as in statistics. Because of the non-experimental nature of almost all economic data, economic models usually involve a large number of inferences. In the linear regression model, if the errors do not follow the assumptions of the classical linear regression model, then test based on this assumption are not appropriate and give misleading result. Different types of violations may be caused due to multicollinearity, heteroscedasticity, autocorrelation, etc. Violation of the assumptions of regression disturbances results in autocorrelation and heteroscedasticity. Therefore, it is highly desirable to be able to perform diagnostic tests of the regression disturbances in the presence of autocorrelation and or heteroscedasticity.

There is an extensive literature on testing of autocorrelation coefficients in the linear regression model, see for example Durbin-Watson (1950), Durbin (1970), Box and Pierce (1970), Wallis (1972), Ljung and Box (1978), Breush and Godfrey (1978) etc. But these tests are two-sided in nature and not suitable for testing one-sided alternatives. To overcome this situation one-sided tests are proposed by Majumder and King (1999), Basak, Rois and Majumder (2005), and Rois, Basak and Majumder (2008) using distance-based approach. Monte Carlo experiments reported by them show that the one-sided tests for autocorrelation rejects the null hypothesis more frequently than usual two-sided tests. Unfortunately, all these one-sided tests are tested autocorrelation coefficients but not the regression coefficients in the model. Thus the problems of nuisance parameters arise in the tests.

Econometric inferences, particularly hypothesis testing, may be affected seriously when nuisance parameters are present in the model. One possible explanation is that the presence of nuisance parameters causes biases in key 
estimates used in the tests. As Durbin and Watson (1971) first showed, this problem of nuisance parameters can be overcome by the use of invariance arguments. A maximal invariant statistic can be expressed as functions of the maximal invariant, optimal invariant testing procedures can be constructed by treating the maximal invariant as the observed data (King \& Hiller, 1985; King, 1987).

In this context, Ara and King (1993) considered treating the standard maximal invariant statistic as the observed data and using its density as the likelihood function. They showed that this is also equivalent to basing the inference on the marginal-likelihood function. Use of the marginal likelihood function first suggested by Kalbfleisch and Sportt (1970). Others too have proposed its use, particularly in the context of estimation, include Levenbach (1972; 1973), Patterson and Thompson (1975), Cooper and Thompson (1977) and Tunnicliffe Waison (1989). The main theme of this literature is that the use of the marginal likelihood helps reduce bias. Results reported by Corduas (1986) and Ara and King (1993) show that the LR and LM tests based on marginal likelihood functions are clearly more accurate than their conventional counterparts. But these tests are also two-sided in nature.

Many econometric models provide us with prior information about some or all of their unknown parameters. Such information usually comes from economic theory, from previous empirical studies or from functional considerations such as variance always being nonnegative. For this reason, many econometric testing problems are potentially either strictly one-sided or partially one-sided. For example, the own price and income elasticity coefficients in demand analysis, and variances of error components in panel data model with individual and time error components can be expected to be positive (Majumder, 1999). In practice, often the amount of data available to conduct a test is limited. In this situation, two-sided tests are no longer valid. So the one-sided and partially one-sided tests may be able to improve the quality of inferences. In such situation, the maximum likelihood based LR test can be improved by using distance-based approach for testing higher order autocorrelation in presence of nuisance parameters. So we expect better power performance of our one-sided maximum likelihood-based LR test.

Our particular focus in this paper is on the use of maximum likelihood-based LR test (Ara \& King, 1993) with distance-based approach (Majumder \& King, 1999) for testing higher order autocorrelation in the linear regression model that involves nuisance parameters. Finally, make a comparative study of one-sided maximum likelihoodbased LR test (DMLR) with one-sided LR (DLR) and usual two-sided LR tests for testing higher order autocorrelation in linear regression model that involves nuisance parameters.

The organization of the paper is as follows. In section 2, we discuss the model and hypothesis uses to develop the test. Our proposed distance-based marginal likelihood one-sided LR (DMLR) test discuss in section 3 . We also express distance-based one-sided LR (DLR) test in section 4. In section 5 we introduce the Monte Carlo simulation. A comparison is made between the powers of DMLR test with one-sided LR test (DLR) and usual two-sided LR test in section 6. Finally, section 7 contains concluding remarks.

\section{Model and Hypothesis}

Consider the following regression model,

$$
y=X \beta+u, u \sim N\left(0, \sigma^{2} \Omega(\theta)\right)
$$

where, $y$ and $u$ are $(n \times 1), \beta$ is $(k \times 1)$ vector of parameters, $X$ is $(n \times k)$, nonstochastic and of rank $k<n$, and $\Omega(\theta)$ is a symmetric $(n \times n)$ matrix that is positive definite for $\theta(p \times 1)$ in a subset of $R^{p}$. Here we assume that the disturbance term follows a stationary $A R(p)$ process,

$$
u_{t}=\rho_{1} u_{t-1}+\rho_{2} u_{t-2}+\ldots+\rho_{p} u_{t-p}+\varepsilon_{t},
$$

where, $\varepsilon_{t} \sim N(0,1)$. Here $\Omega$ is a function of unknown parameter(s) $\theta$, where, $\theta=\left(\sigma_{\varepsilon}^{2}, \hat{\rho}\right)$ and $\Omega(\theta) \neq \sigma^{2} I$. In this context, whenever only the error components $\rho$ are tested to check the validity of certain assumption, then the regression coefficients $\beta$ become the nuisance parameters for the test.

We are interested in testing,

$$
H 1 \quad H_{0}: \rho=0 \text {, against } H_{1}: \rho>0,
$$

where, $\rho=\left(\rho_{1}, \rho_{2}, \ldots, \rho_{p}\right)^{\prime}$ is a $(\rho \times 1)$ matrix. The alternative hypothesis is called strictly one-sided, in such situation, usual two-sided LR test is not totally suitable. In this testing process, we have tested the autocorrelation of the error terms but not the slope and intercept parameters. So our testing procedure contains nuisance parameters. As a result distance-based one-sided DLR test is affected by nuisance parameters. The effect of the nuisance parameters can be eliminated by the marginal likelihood. Hence, we proposed a distance-based maximum likelihood one-sided LR (DMLR) test. 
In this paper we developed strictly one-sided DMLR test by considering the linear regression model (2.1) with the disturbance term $u$ follows $A R(p)$ process (2.2) for the alternative hypothesis $H 1$.

\section{Distance-based Marginal Likelihood One-sided LR (DMLR) Test}

Now we are in the position to derive the test statistic of our proposed distance-based marginal likelihood one-sided LR (DMLR) test. To develop the DMLR test statistic we need a clear knowledge about the marginal-likelihood based test (Ara \& King, 1993; Ara, 1995) and distance-based approach (Majumder, 1999).

\subsection{Distance-based Approach}

Distance-based approach suggests that we have to determine whether the estimated parameters under test likely to be closer to null hypothesis or to alternative hypothesis. Majumder's (1999) approach is outlined below for general testing problem: Suppose we are interested in testing a hypothesis of a parametric model in which the parameter of interest, $\theta$, is restricted under the alternative hypothesis. More specifically, we wish to test

$$
H_{0}: \theta=0, \text { versus } H_{a}: \theta>B
$$

based on the $(n \times 1)$ random vector $y$ whose distribution has probability density function $f(y, \theta)$, where $\theta \in R^{p}$ is a subvector of an unknown parameter $\Theta \in R^{s}$ ) and $B$ is a subset of $R^{p}$. Let $\theta$ be a suitable estimate of $\theta$ such that $\theta$ is asymptotically distributed as normal with variance-covariance matrix $c I^{-1}(\theta)$ where $c$ is a constant and $I(\theta)$ is the information matrix. Following Shapiro (1988), Kodde and Palm's (1986) and Majumder (1999) suggest that we should determine the closest point in the maintained hypothesis from the unconstrained point. This closest point is the solution of the following distance function or optimal function in the metric $c I^{-1}(\theta)$ of the parameter vector $\hat{\theta}$,

$$
\|\breve{\theta}-\hat{\theta}\|^{2}=(\breve{\theta}-\hat{\theta})^{\prime} I(\theta)(\breve{\theta}-\hat{\theta}), \quad \text { subject to } \quad \breve{\theta} \in B .
$$

The closest point or optimized $\breve{\theta}$ can be used in any appropriate two-sided tests to obtain the corresponding distancebased one-sided and partially one-sided tests. The asymptotic null hypothesis distribution generally follows a mixture of the corresponding two-sided distributions (Majumder, 1999).

\subsection{Marginal Likelihood LR (MLR) Test}

Ara and King (1993) and Ara (1995) developed marginal likelihood based LR test for testing regression disturbances in linear regression model that involves nuisance parameters. Here we express the summary information of marginal likelihood based LR test for exploring the concept of proposed distance-based marginal likelihood one-sided LR (DMLR) test. Under (2.2), $u \sim N\left(0, \sigma^{2} \Omega(\theta)\right)$ where $u$ has the stable variance-covariance matrix,

$$
\Omega(\theta)=\left[L_{1}^{\prime} L_{1}-N N^{\prime}\right]^{-1},
$$

(Ljung \& Box, 1979) in which $L_{1}$ is the $n \times n$ matrix

$$
L_{1}=\left(\begin{array}{ccccccc}
1 & 0 & \cdots & & & \cdot & 0 \\
-\theta_{1} & 1 & \cdots & & & & 0 \\
\vdots & \vdots & \vdots & \vdots & \vdots & \vdots & \vdots \\
-\theta_{p} & -\theta_{p-1} & \cdots & & & & \cdot \\
0 & -\theta_{p} & \cdots & & & & \cdot \\
\vdots & \vdots & \vdots & \vdots & \vdots & \vdots & \vdots \\
. & . & \cdots & . & \cdots & 1 & 0 \\
0 & 0 & \cdots & -\theta_{p} & \cdots & -\theta_{1} & 1
\end{array}\right),
$$

and $N$ is the $n \times p$ matrix of zeros but with the top $p \times p$ block being

$$
\left(\begin{array}{ccccc}
-\theta_{p} & -\theta_{p-1} & -\theta_{p-2} & \cdots & -\theta_{1} \\
0 & -\theta_{p} & -\theta_{p-1} & \cdots & -\theta_{2} \\
0 & 0 & -\theta_{p} & \cdots & \cdot \\
\vdots & \vdots & \vdots & \vdots & \vdots \\
0 & 0 & 0 & \cdots & -\theta_{p}
\end{array}\right) .
$$


From (3.2.1), Ara (1995) deduces the inverse of the Cholesky decomposition of $\Omega(\theta)$, which we denote by $H(\theta)=$ $\Omega(\theta)^{-\frac{1}{2}}$, is equal to $L_{1}$ but with the top left $p \times p$ block replaced by the lower triangular matrix,

$$
\left(\begin{array}{ccccc}
h_{11} & 0 & 0 & \cdots & 0 \\
h_{21} & h_{22} & 0 & \cdots & 0 \\
h_{31} & h_{32} & h_{33} & \cdots & 0 \\
\vdots & \vdots & \vdots & \vdots & \vdots \\
h_{p 1} & h_{p 2} & h_{p 3} & \cdots & h_{p p}
\end{array}\right) .
$$

Where the $h_{i j}$ values calculated recursively in the order indicated:

$$
\begin{gathered}
h_{p, p}=\left(1-\theta_{p}^{2}\right)^{\frac{1}{2}} \\
h_{p, p-i}=\left(-\theta_{i}-\theta_{p-i} \theta_{p}\right) / h_{p p}, \quad i=1, \ldots, p-1
\end{gathered}
$$

For $q=p-1, p-2, \ldots, 2$ and $m=p-q$,

$$
\begin{gathered}
h_{q, q}=\left(1+\sum_{i=1}^{q-1} \theta_{i}^{2}-\sum_{i=m+1}^{p} \theta_{i}^{2}-\sum_{i=1}^{m} h_{q+i, q}^{2}\right)^{\frac{1}{2}} \\
h_{q, q-k}=\left(-\theta_{k}-\sum_{i=1}^{q-k-1} \theta_{i} \theta_{i+k}-\sum_{i=m+1}^{p-k} \theta_{i} \theta_{i+k}-\sum_{i=q+1}^{p} h_{i, q} h_{i, q-k}\right) / h_{q q},
\end{gathered}
$$

for $k=1, \ldots, q-2$ and

$$
h_{q, 1}=\left(-\theta_{q-1}-\theta_{m+1} \theta_{p}-\sum_{i=q+1}^{p} h_{i, q} h_{i, 1}\right) / h_{q q}
$$

Finally,

$$
h_{11}=\left(1-\theta_{p}^{2}-\sum_{i=1}^{p-1} h_{i+1,1}^{2}\right)^{\frac{1}{2}} .
$$

The LR test of $H_{0}$ requires maximum likelihood estimates of $\theta$. This involves maximizing

$$
\log \left(\prod_{i=1}^{p} h_{i i}\right)-\frac{1}{2} \log \left|X^{*^{\prime}} X^{*}\right|-\frac{m}{2} \log \left(e^{\prime} e\right)
$$

with respect to $\theta$, where $X^{*}=H(\theta) X$ and $e$ is the OLS residual vector from regression

$$
H(\theta) y=H(\theta) X+H(\theta) u .
$$

Let $\hat{\theta}$ denote the value of $\theta$ which maximizes (3.2.2) and let $\hat{h_{i j}}$ and $\hat{X}^{*}$ denote $h_{i j}$ and $X_{*}$, respectively, evaluated at $\theta=\hat{\theta}$. Furthermore, let $\hat{e}$ denote the OLS residuals from (3.2.3) with $\theta=\hat{\theta}$.

Thus the convenient from of the marginal-likelihood-based LR test statistic developed by Ara (1995) is

$$
M L R=\log \left|X^{\prime} X\right|+\log \left(\prod_{i=1}^{p} h_{i i}\right)-\log \left|X^{*^{\prime}} X^{*}\right|-m \log \left(\frac{e^{\prime} e}{z^{\prime} z}\right),
$$

under $H_{0}$, (3.2.4) has an asymptotic $\chi_{p}^{2}$ distribution (Ara, 1995).

\subsection{Distance-based Marginal Likelihood One-sided LR (DMLR) Test}

In our distance-based marginal likelihood one-sided LR (DMLR) test we first estimate the optimum values of $\hat{\beta}$ and $\hat{\rho}$ according to the general formulation of distance-based approach, subject to the restrictions, $H 1$. Now the DMLR test statistic becomes,

$$
D M L R=\log \left|X^{\prime} X\right|+2 \log \left(\prod_{i=1}^{p} \tilde{h_{i i}}\right)-\log \left|\tilde{X^{*^{\prime}}} \tilde{X}^{*}\right|-\operatorname{mlog}\left(\frac{\tilde{e^{\prime}} \tilde{e}}{\tilde{z^{\prime}} \tilde{z}}\right),
$$


where, $\tilde{h_{i i}}, \tilde{X^{*}}, \tilde{e}$ and $\tilde{z}$ are the optimized value of $h_{i i}, X^{*}, \hat{e}$ and $\hat{z}$, respectively, using the optimized value of $\beta$ and $\tilde{\rho}$ subject to the restrictions, $H 1$. Under the null hypothesis the distribution of the test statistic (3.3.1) follows asymptotically weighted mixture of chi-square distribution with $p$ degrees of freedom (Kodde \& Palm, 1986; Shaprio, 1988; Majumder, 1999).

\section{Distance-based One-sided LR Test}

The likelihood ratio (LR) test requires calculation of both restricted and unrestricted estimators. If both are simple to compute, this will be a convenient way to proceed. The general form of two-sided LR statistic is,

$$
L R=2\left(l\left(\hat{\theta_{a}}\right)-l\left(\hat{\theta_{0}}\right)\right.
$$

where, $l\left(\hat{\theta_{0}}\right)$ and $l\left(\hat{\theta_{a}}\right)$ are the unrestricted and restricted maximized log-likelihood functions, respectively. The asymptotic null hypothesis distribution of (4.1) follows a central chi-square distribution with $k$ degrees of freedom. But the two-sided LR test is not appropriate when the alternative hypothesis becomes strictly one-sided. In distance-based LR test we have to estimate the optimum values of $l\left(\hat{\theta_{0}}\right)$ and $l\left(\hat{\theta_{a}}\right)$ according to the general formulation of distance-based approach (Majumder, 1999; Basak et al., 2005; Rois et al., 2008), subject to the restriction $H 1$, discussed in section 3.1. Then the statistic becomes,

$$
D L R=\breve{L R}=2\left(l\left(\breve{\theta_{a}}\right)-l\left(\breve{\theta_{0}}\right)\right)
$$

where, $l\left(\breve{\theta_{0}}\right)$ is the unrestricted optimized value and $l\left(\breve{\theta_{a}}\right)$ is the optimized value subject to the restrictions, $H 1$.

\section{Monte Carlo Simulation}

Monte Carlo simulations are carried out to compare the powers of the usual two-sided LR test, DLR test and the DMLR test for detecting higher order autocorrelation of a regression model of the form (2.1). Here, we use real and artificially generated explanatory variables $(X)$. In order to carry out Monte Carlo simulation we generate the following second order autocorrelated disturbance terms respectively,

$$
u_{t}=\rho_{1} u_{t-1}+\rho_{2} u_{t-2}+\varepsilon_{t},
$$

where, $\varepsilon_{t} \sim N(0,1)$, and consequently we generate the model (2.1). We perform 5,000 replications to calculate (size corrected) simulated powers of the new and existing tests, when the error term follows second order autoregressive scheme (5.1).

\subsection{Experimental Design}

In order to compare the power properties of proposed DMLR test with one-sided DLR test and usual two-sided LR test we use two different types of design matrices-real data. We use the following design matrices:

D1: A constant dummy and the per-capita income in constant dollars, average price for the single-family rate tariff electricity consumption in Summer, electricity consumption in winter of the San Diego Gas and Electric Company for the period 1972-1993 (Ramanathan, 1995, 502-503).

D2: A constant dummy and the quarterly Australian consumer price index covering the period 1959-1979, lagged one quarter of the variable.

For testing the maintained hypothesis, all the design matrices D1 and D2 are employed. The following matrices are used in the experiment.

$\mathrm{X} 1$ : A constant dummy and all (four) real variables of D1,

X2: A constant dummy and all (two) real variables of D2.

We can perform our experiment for different values of the parameters $\rho_{i}, i=1,2, \ldots, p$ (0 to 0.9$)$. For the secondorder autoregressive scheme (5.1) we estimate simulated powers for testing one-sided hypothesis (2.3) and the above X1, X2 matrices for $n=50$. Here we use selected values of $\rho_{1}$ and $\rho_{2}=0(0.2) 0.8$.

\section{Results}

This section compares the powers of the existing two-sided LR test, one-sided DLR test and the newly proposed DMLR tests for testing $H 1$, in the context of linear regression model (2.1), which involves nuisance parameters. We use four regressors for the first set, which is per capita income, average price of the single-family rate tariff, electricity consumption in summer, electricity consumption in winter of the San Diego Gas and Electric Company 
for the period 1972-1993, (Ramanathan, 1995). In the second set we use two explanatory variables of lagged data for testing the hypothesis. The estimated simulated powers of these tests are presented in Appendix, Tables 1-2 with Figures 1-2 for the design matrices defined in section 5.1. These tables represent the simulated powers of DMLR test along with one-sided DLR and two-sided LR tests for second order autoregressive scheme (5.1), when the alternative hypothesis is of the form $H_{1}$. In both tables, the estimated sizes of the three tests is 0.05 against $A R(2)$ disturbances when asymptotic critical values at five percent nominal level are used. Thus all the tests have size-corrected power.

Table 1. The powers of the DMLR, DLR and LR tests of hypothesis $H_{1}$, for second-order autoregressive scheme, using stationary time series data and quarterly data on electricity demand for 50 observations

\begin{tabular}{|c|c|c|c|c|}
\hline \multirow{2}{*}{$\rho_{1}$} & \multirow{2}{*}{$\rho_{2}$} & \multicolumn{3}{|c|}{$\begin{array}{c}k=4 \\
X 1 \\
\left(R^{2}=0.363\right)^{*}\end{array}$} \\
\hline & & DMLR & DLR & LR \\
\hline \multirow{5}{*}{0} & 0 & 0.05 & 0.05 & 0.05 \\
\hline & 0.2 & 0.283 & 0.280 & 0.119 \\
\hline & 0.4 & 0.738 & 0.735 & 0.481 \\
\hline & 0.6 & 0.970 & 0.969 & 0.891 \\
\hline & 0.8 & 0.999 & 0.999 & 0.993 \\
\hline \multirow{5}{*}{0.2} & 0 & 0.288 & 0.258 & 0.101 \\
\hline & 0.2 & 0.526 & 0.486 & 0.193 \\
\hline & 0.4 & 0.830 & 0.816 & 0.544 \\
\hline & 0.6 & 0.970 & 0.968 & 0.866 \\
\hline & 0.8 & 0.996 & 0.996 & 0.978 \\
\hline \multirow{5}{*}{0.4} & 0 & 0.772 & 0.700 & 0.44 \\
\hline & 0.2 & 0.884 & 0.851 & 0.631 \\
\hline & 0.4 & 0.957 & 0.946 & 0.839 \\
\hline & 0.6 & 0.985 & 0.983 & 0.923 \\
\hline & 0.8 & 1 & 1 & 1 \\
\hline \multirow{5}{*}{0.6} & 0 & 0.982 & 0.960 & 0.857 \\
\hline & 0.2 & 0.988 & 0.983 & 0.923 \\
\hline & 0.4 & 0.988 & 0.986 & 0.949 \\
\hline & 0.6 & 1 & 1 & 1 \\
\hline & 0.8 & 1 & 1 & 1 \\
\hline
\end{tabular}

${ }^{*}$ Multicollinearity Exits

Table 1 reveals the estimated powers of one-sided marginal likelihood LR (DMLR), one-sided LR (DLR) and two-sided LR tests for $n=50$ and design matrix $X 1$, which contains four explanatory independent variables with problem of multicollinearity. We observe that the powers of DMLR test are slightly higher than one-sided DLR test but significantly greater than two-sided LR test near null value. For example, the power of the DMLR test and DLR, LR tests are $0.283,0.280$ and 0.110 , respectively, for $\rho_{1}=0.0, \rho_{2}=0.2, k=4$ and $n=50$. 


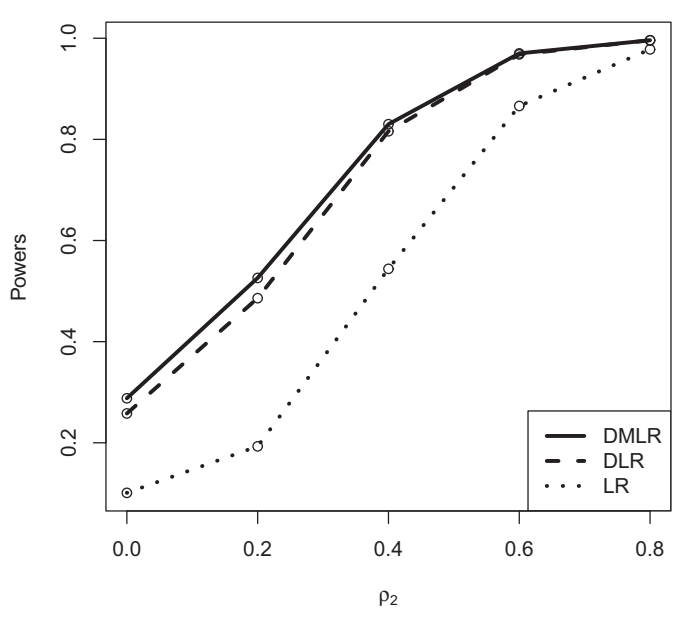

Figure 1. Power curves of DMLR, DLR and LR tests of hypothesis $H_{1}$, when $n=50, k=4, p=2$ and fixed

$$
\rho_{1}=0.2
$$

Typically, powers of all tests are increases as increases. For example, the power of the DMLR test and DLR, LR tests are $0.884,0.851$ and 0.631 , respectively, for $\rho_{1}=0.4$ and $\rho_{2}=0.2$. Figure 1 explores this comparison. For example, the power of the DMLR test and DLR, LR tests are 0.83, 0.816 and 0.544 , respectively, for $\rho_{1}=0.2$, $\rho_{2}=0.4, k=4$ and $n=50$ in Table 1 . In order to investigate the power performances of DMLR test along with DLR, LR tests in the case of lagged data we use X2-matrix, which contains two explanatory variables for testing the hypothesis $H 1$. Table 2 presents the simulated powers of DMLR test, DLR test and LR test.

Table 2. The powers of the DMLR, DLR and LR tests of hypothesis $H_{1}$, for second-order autoregressive scheme, using lagged data

\begin{tabular}{|c|c|c|c|c|}
\hline \multirow{2}{*}{$\rho_{1}$} & \multirow{2}{*}{$\rho_{2}$} & \multicolumn{3}{|c|}{$\begin{array}{c}k=2 \\
X 2 \\
r=0.999)\end{array}$} \\
\hline & & DMLR & DLR & LR \\
\hline \multirow{5}{*}{0} & 0 & 0.05 & 0.05 & 0.05 \\
\hline & 0.2 & 0.305 & 0.301 & 0.135 \\
\hline & 0.4 & 0.777 & 0.772 & 0.527 \\
\hline & 0.6 & 0.982 & 0.981 & 0.921 \\
\hline & 0.8 & 1 & 1 & 0.996 \\
\hline \multirow{5}{*}{0.2} & 0 & 0.277 & 0.264 & 0.102 \\
\hline & 0.2 & 0.547 & 0.515 & 0.205 \\
\hline & 0.4 & 0.868 & 0.853 & 0.587 \\
\hline & 0.6 & 0.979 & 0.979 & 0.897 \\
\hline & 0.8 & 0.998 & 0.998 & 0.985 \\
\hline \multirow{5}{*}{0.4} & 0 & 0.759 & 0.714 & 0.446 \\
\hline & 0.2 & 0.897 & 0.876 & 0.660 \\
\hline & 0.4 & 0.964 & 0.959 & 0.862 \\
\hline & 0.6 & 0.982 & 0.981 & 0.917 \\
\hline & 0.8 & 1 & 1 & 1 \\
\hline \multirow{5}{*}{0.6} & 0 & 0.981 & 0.968 & 0.876 \\
\hline & 0.2 & 0.987 & 0.985 & 0.938 \\
\hline & 0.4 & 0.988 & 0.987 & 0.946 \\
\hline & 0.6 & 1 & 1 & 1 \\
\hline & 0.8 & 1 & 1 & 1 \\
\hline
\end{tabular}


We observe from the Table 2 that the simulated power of the DMLR test is higher than DLR and LR tests in all cases. For example, the simulated powers of the DMLR, one-sided DLR and LR tests are 0.981, 0.968 and 0.876, respectively, for $\rho_{1}=0.6, \rho_{2}=0.0, k=2$ and $n=50$ in Table 2 .

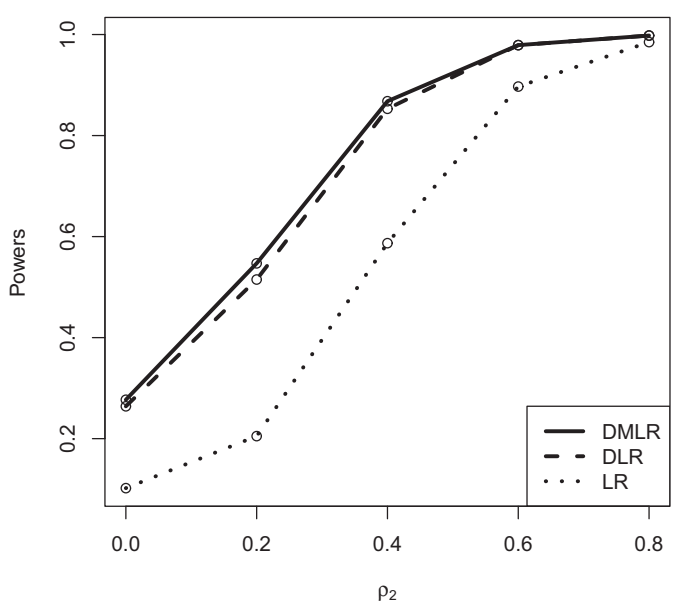

Figure 2. Power curves of DMLR, DLR and LR tests of hypothesis $H_{1}$, when $n=50, k=2, p=2$ and fixed

$$
\rho_{1}=0.2
$$

There is a clear tendency for DMLR test to be more powerful for larger values of $\rho$. For example, the simulated powers of the DMLR test, one-sided DLR and LR tests are 0.547, 0.515 and 0.205, respectively, for $\rho_{1}=0.2$, $\rho_{2}=0.2, k=2$ and $n=50$. Figure 2 presents similar dominance nature of DMLR test over one-sided DLR test and two-sided LR test.

We observe from Monte Carlo simulation study that in all cases, i.e. correlated, uncorrelated or even when multicollinearity exists, our proposed DMLR test gives higher power than two-sided LR test and one-sided DLR test. All the figures and tables represent that the simulated power of distance-based marginal likelihood one-sided DMLR test is always superior to the usual two-sided LR test and even also to the distance-based one-sided DLR test.

In summary, the use of marginal-likelihood based test rather than their traditional counterparts does result typically in a more accurate test in terms of power. These improvements are very clear-cut in all the cases. All this evidence and the findings do suggest there can be clear advantages in using one-sided marginal likelihood based LR tests in place of one-sided DLR and two-sided LR tests for testing higher order autocorrelation in linear regression model which involves nuisance parameters.

\section{Conclusions}

This paper develops distance-based marginal likelihood one-sided DMLR test for testing higher order autocorrelations of the disturbances in a linear regression model for the one-sided alternatives in the presence of nuisance parameter(s). In this study, regression coefficients are not of the interest and that result in nuisance parameters. Since, the presence of nuisance parameters causes unwanted complications in statistical and econometric inference procedures. So we expect that eliminating the influence of nuisance parameters from the test will improve our test performance. Monte Carlo results indicate similar message that newly proposed distance-based marginal likelihood one-sided LR (DMLR) test performs better than one-sided LR and two-sided LR tests in all cases discussed in this paper.

\section{References}

Ara, I. (1995). Marginal Likelihood based tests of regression disturbances. An unpublished thesis, Monash University, Australia.

Ara, I., \& King, M. L. (1993). Marginal likelihood based tests of a subvector of the parameter vector of linear regression disturbances. In C. S. Forbes, P. Kofman and T. R. L. Fry (eds.), Proceedings of the Econometrics 
Conference at Monash University, Monash University, 69-106.

Basak, T., Rois, R., \& Majumder, A. K. (2005). One-Sided Version of the Breusch-Godfrey test for Testing Higher-Order Autocorrelation - A Distance-Based Approach. Proceedings of the Two One-Day Seminars, Bangladesh Statistical Association, Dhaka.

Breusch, T. S. (1978). Testing for autocorrelation in dynamic linear models. Australian Economic Papers, 17, 334-355. http://dx.doi.org/10.1111/j.1467-8454.1978.tb00635.x

Box, G. E. P., \& Pierce, D. A. (1970). Distribution of the Autocorrelations in Autoregressive Moving Average Time Series Models. Journal of American Statistical Association, 65, 1509-1526. http://dx.doi.org/10.1080/01621459.1970.10481180

Cooper, D. M., \& Thompson, R. (1977). A note on the estimation of parameters of the autoregressive-moving average process. Biometrika, 64, 625-628.

Corduas, M. (1986). The use of marginal likelihood in testing for serial correlation in time series regression, an unpublished M. Phil., Thesis, University of Lancaster.

Durbin, J., \& Watson, G. S. (1950). Testing for serial correlation in least squares regression I. Biometrika, 37, 409-428.

Durbin, J. (1970). Testing for serial correlation in least squares regression when some of the regressors are lagged dependent variables. Econometrica, 38, 410-421. http://dx.doi.org/10.2307/1909547

Durbin, J., \& Watson, G. S. (1971). Testing for Serial Correlation in Least Squares Regression III. Biometrika, 58, $1-19$.

Godfrey, L. G. (1978). Testing for higher order serial correlation in regression equations when the regressors include lagged dependent variables. Econometrica, 46, 1303-1310. http://dx.doi.org/10.2307/1913830

Kalbfleisch, J. D., \& Sprott, D. A. (1970). Application of Likelihood methods to models involving large numbers of parameters. Journal of the Royal Statistical Society, Ser. B, 32, 175-208.

King, M. L. (1987). Testing for autocorrelation in linear regression models: A survey, in M. L. King and D. E. A. Giles (eds.). Specification Analysis in linear Model (pp. 19-73). London, England: Routledge and Kegan paul.

King, M. L., \& Hillier, G. H. (1985). Locally best invariant tests of the error covariance matrix of the linear regression model. Journal of the Royal Statistical Society, Ser. B, 47, 98-102.

Kodde, D. A., \& Palm, F. C. (1986). LR criteria for jointly testing equality and inequality restrictions. Econometrica, 54, 1243-1248. http://dx.doi.org/10.2307/1912331

Levenbach, H. (1972). Estimation of autoregressive parameters from a marginal likelihood function. Journal of the American Statistical Association, 59, 61-71.

Levenbach, H. (1973). Estimating heteroscedasticity from a marginal likelihood function. Journal of the American Statistical Association, 68, 436-439. http://dx.doi.org/10.1080/01621459.1973.10482451

Ljung, G. M., \& Box, G. E. P. (1978). On a measure of lack of fit in time series. Biometrika, 65, $297-303$. http://dx.doi.org/10.1093/biomet/65.2.297

Ljung, G. M., \& Box, G. E. P. (1979). On a measure of lack of fit in time series models. Biometrika, 66, $265-270$. http://dx.doi.org/10.1093/biomet/66.2.265

Majumder, A. K. (1999). One-sided and partially one-sided hypothesis testing in econometrics. An unpublished thesis, Monash University, Australia.

Majumder, A. K., \& King, M. L. (1999). One-sided test of multiple inequality constraints-a distance-based approach, Paper presented at the Econometric Society Australasian Meeting 7-10 July. Canberra.

Patterson, H. D., \& Thompson, R. (1975). Maximum likelihood estimation of components of variance, in L. C. A. Corsten and T. Postelnicu (eds.). Proceedings of the 8th international Biometric Conference. Academy of Socialist Republic of Rumania, Bucharest.

Ramanathan, R. (1995). Introductory Econometrics with applications (3rd ed., pp. 502-503). New York: Harcourt 
College Publishers.

Rois, R., Basak, T., \& Majumder, A. K. (2008). On the Test of Restricted Higher Order Autocorrelation: A Wald-Type Distance-Based Approach. Jahangirnagar University Journal of Science, 31(1), 127-142.

Shapiro, A. (1988). Towards a unified theory of inequality constrained testing in multivariate analysis. International Statistical Review, 56, 49-62. http://dx.doi.org/10.2307/1403361

Tunnicliffe Wilson, G. (1989). On the use of marginal likelihood in time series model estimation. Journal of the Royal Statistical Society, Ser. B, 51, 15-27.

Wallis, K. F. (1972). Testing for fourth order autocorrelation in Quarterly regression equations. Econometrica, 40 617-636. http://dx.doi.org/10.2307/1912957 